\title{
Complete Postsurgical Left Ventricular-Aortic Discontinuity and Pseudoaneurysm Formation
}

\author{
Weibo $\mathrm{Fu}^{1}$, Paran Davari ${ }^{2}$, Hossam Alslaim ${ }^{1}$, Niayesh Cortese ${ }^{1}$, Mohamed Issa ${ }^{3}$, William \\ Bates $^{4}$, Victor Ferraris ${ }^{3}$, and Michael Winkler ${ }^{4}$ \\ ${ }^{1}$ Medical College of Georgia \\ ${ }^{2}$ University of California San Francisco School of Medicine \\ ${ }^{3}$ University of Kentucky Medical Center \\ ${ }^{4}$ Augusta University Medical College of Georgia
}

January 18, 2022

\begin{abstract}
Background: Left ventricular outflow tract pseudoaneurysm is a rare but potentially fatal complication of aortic valve replacement, infective endocarditis, and suture dehiscence. Left ventricular-aortic discontinuity is a severe and uncommon manifestation of IE. For patients who have a long-standing history of endocarditis, peri-annular lesions in the aortic valve may rupture, leading to the rare occurrence of complete, or total, left ventricular-aortic discontinuity. Methods: We present a case of complete postoperative left ventricular-aortic discontinuity and massive circumferential left ventricular outflow tract pseudoaneurysm discovered during a 3-month follow-up visit. Results: Post-operative cardiac CT of a patient demonstrated dehiscence of a recently placed surgical aortic valve from the left ventricular outflow tract, with massive circumferential pseudoaneurysm formation. Only a small remnant of the membranous interventricular septum connected the aortic root to the heart, informing the diagnosis of complete left ventricular-aortic discontinuity. Conclusion: The clinical presentation of a left ventricular outflow tract pseudoaneurysm with concomitant left ventricular-aortic discontinuity is commonly nonspecific or clinically silent; thus, it requires a high index of suspicion and use of multimodality imaging for diagnosis and management.
\end{abstract}

\section{Introduction:}

Left ventricular outflow tract (LVOT) pseudoaneurysm is a rare but potentially fatal complication of aortic valve replacement (AVR), infective endocarditis (IE), and suture dehiscence. Since AVR is the established treatment for aggressive IE with septic emboli, such patients who undergo the procedure have an increased summative risk of this complication. ${ }^{1}$ Like LVOT pseudoaneurysm, left ventricular-aortic (LV-Ao) discontinuity is a severe and uncommon

manifestation of IE. For patients who have a long-standing history of endocarditis, peri-annular lesions in the aortic valve may rupture, leading to LV-Ao discontinuity. Additionally, after radical debridement for extensive IE, patients may present with iatrogenic LV-Ao discontinuity. In either case, LV-Ao discontinuity has high morbidity and mortality if left untreated. ${ }^{2}$

Case Report:

A 29-year-old male with a past medical history of intravenous drug abuse and hepatitis C was transferred from an outside hospital for evaluation of fever, heart murmur, and concerns for infective endocarditis. On arrival, the patient had mild chest pain and dyspnea. Blood cultures were positive for Serratia marcescens. Transthoracic echocardiogram (TTE) revealed large vegetations on the aortic valve with severe aortic regurgitation. His hospital course was complicated by acute encephalopathy and an acute left middle cerebral 
artery territory embolic stroke. The patient completed a 6-week course of antibiotics and underwent an uncomplicated aortic valve replacement with a $23 \mathrm{~mm}$ Magna Ease bioprosthetic valve. TTE prior to discharge showed a normal functioning prosthetic valve, with normal left ventricular size and function. Although initially symptom free, the patient began experiencing attacks of transient dizziness two weeks before a scheduled 3-month follow-up visit. A two-dimensional transthoracic echocardiogram was obtained demonstrating severe aneurysmal change within the aortic root (Figure 1). Ejection fraction was $55 \%$ and showed no evidence of aortic regurgitation. Peak instantaneous pressure gradient across the prosthetic valve was 7 $\mathrm{mmHg}$ with a mean pressure gradient of $4 \mathrm{mmHg}$. A cardiac CT was performed using a Somatom Force Scanner (Siemens, Erlangen, Germany). Images were reconstructed from the end diastolic phase using a soft vascular kernel and a model based iterative algorithm utilizing a small field of view and interpolative techniques to achieve a fine spatial resolution. Image post processed was performed with an advanced image postprocessing server (Aquarius Intuition, Terarecon, Foster City, CA).

The cardiac CT revealed complete dehiscence of the surgical valve from the LVOT, with a gap of $2 \mathrm{~cm}$, and a massive circumferential pseudoaneurysm. A ribbonlike remnant of the membranous interventricular septum was all that tethered the aortic root to the heart. The coronary arteries were stretched but not compressed by the pseudoaneurysm. Fortuitously, there was no communication with the right ventricle or atrium. The bioprosthetic aortic valve leaflets, surprisingly, appeared normal. (Figure 2) Volume rendering with blood pool inversion technique allowed for delineation of the pseudoaneurysm's boundaries and its relation to the aortic prosthetic valve, atria, ventricles, and pulmonary veins. (Figure 3 and Cine.1)

While initially reluctant, the patient opted for surgical reintervention during a one month follow up visit after a repeat TTE revealed a new anterior wall motion abnormality assumed to be due to effacement of the lumen of the left main coronary artery. Repeat surgery required extensive debridement and replacement of the Magna Ease pericardial prosthesis with a Medtronic Freestyle porcine root. Following the surgery, the anterior wall motion abnormality resolved, and the patient was transported to the intensive care unit in stable condition.

Discussion:

Over its clinical course, IE can destroy large areas of tissue in the aortic annulus, reducing the strength and stability of native cardiac tissue while simultaneously increasing the risk of LV-Ao discontinuity. Extensive debridement of infected cardiac structures prior to valve replacement further adds to risk of discontinuity because it decreases the amount of available healthy tissue available for restructuring and repair of the LVOT. ${ }^{3}$ The avascular nature of the mitral aortic intervalvular fibrosa (MAIVF) in the LVOT makes it susceptible to infection and abscess formation in the setting of aortic valve and root endocarditis. Aortic valve regurgitation in the setting of aortic valve endocarditis may also contribute to MAIVF pseudoaneurysm formation, as the infected regurgitation jets hit the inherently susceptible MAIVF. In the setting of IE, Staphylococcus species are the most implicated cause of abscess and pseudoaneurysm formation. ${ }^{4}$ After surgical correction, follow up by TTE can assess prosthetic valve function but may be insufficient to diagnose new abscesses or pseudoaneurysms. In such cases, other imaging modalities, such as ECG-gated CT or magnetic resonance imaging, can improve sensitivity and accuracy in diagnosis of these complications. As LVOT pseudoaneurysms and complete discontinuity may present with nonspecific signs and symptoms such as heart failure and chest pain, advanced cross section imaging should be considered early. ${ }^{5,6}$ LVOT pseudoaneurysms carry high risk of rupture and subsequent sudden cardiac death with a frequency of up to $50 \%$. TTE has been shown to be an ineffective modality for diagnosing LVOT pseudoaneurysm and discontinuity. ${ }^{4,5,7,8}$ In a previously published review, definitive diagnoses by TTE were achieved only in $36 \%$ of cases and missed up to $65 \%$ of the time. ${ }^{4}$ Transesophageal echocardiography can increase the sensitivity and specificity for diagnoses of such lesions. Cardiac computed tomography, as exemplified in this case, provides higher sensitivity than TTE and better 3-dimensional assessment of the lesion. ${ }^{4,5}$ 


\section{References:}

1. Habib G, Hoen B, Tornos P, et al. Guidelines on the prevention, diagnosis, and treatment of infective endocarditis (new version 2009): the Task Force on the Prevention, Diagnosis, and Treatment of Infective Endocarditis of the European Society of Cardiology (ESC). Endorsed by the European Society of Clinical Microbiology and Infectious Diseases (ESCMID) and the International Society of Chemotherapy (ISC) for Infection and Cancer. Eur Heart J. 2009;30(19):2369-2413. doi:10.1093/eurheartj/ehp285

2. Uchida T, Kuroda Y, Kobayashi K, Sadahiro M. Surgical treatment for left ventricular-aortic discontinuity and Gerbode defect with endocarditis. Interact Cardiovasc Thorac Surg.

2020;30(3):439-442. doi:10.1093/icvts/ivz284

3. Filippone G, Calia C, Finazzo M, Fazzari F, Caruana G, Argano V. Modified Danielson

Technique for Prosthetic Aortic Valve Endocarditis and Aortoventricular Discontinuity. Tex

Heart Inst J. 2020;47(2):117-120. doi:10.14503/THIJ-17-6506

4. Şahan E, Gül M, Şahan S, Sokmen E, Guray YA, Tufekçioglu O. Pseudoaneurysm of the mitralaortic intervalvular fibrosa. A new comprehensive review. Herz. 2015;40 Suppl 2:182-189.

doi:10.1007/s00059-014-4185-Z

5. Sudhakar S, Sewani A, Agrawal M, Uretsky BF. Pseudoaneurysm of the mitral-aortic intervalvular fibrosa (MAIVF): A comprehensive review. J Am Soc Echocardiogr.

2010;23(10):1009-1112. doi:10.1016/j.echo.2010.07.0159

6. Urmeneta Ulloa J, Galache Osuna JG, Molina Borao I, Sánchez-Rubio Lezcano J. Compression of the left coronary trunk by pseudoaneurysm of the mitral-aortic intervalvular fibrosa. Percutaneous treatment: which to treat first, the trunk or the pseudoaneurysm?. EuroIntervention.2018;13(15):e1814-e1815. Published 2018 Feb 2. doi:10.4244/EIJ-D-16-01042

7. Acioli Pereira L, Fontes Gontijo P, Alcântara Farran J, Palandri Chagas AC, Romano ER, Bento de Souza LC. Giant pseudoaneurysm of the left ventricular outflow tract: a rare disease. Rev Port Cardiol. 2013;32(6):541-544. doi:10.1016/j.repc.2012.11.006

8. Chen F, Wei S, Xiong L, Liu F. Post-traumatic left ventricular outflow tract pseudoaneurysm. Ann Thorac Surg. 2014;97(1):311-312. doi:10.1016/j.athoracsur.2013.04.137 

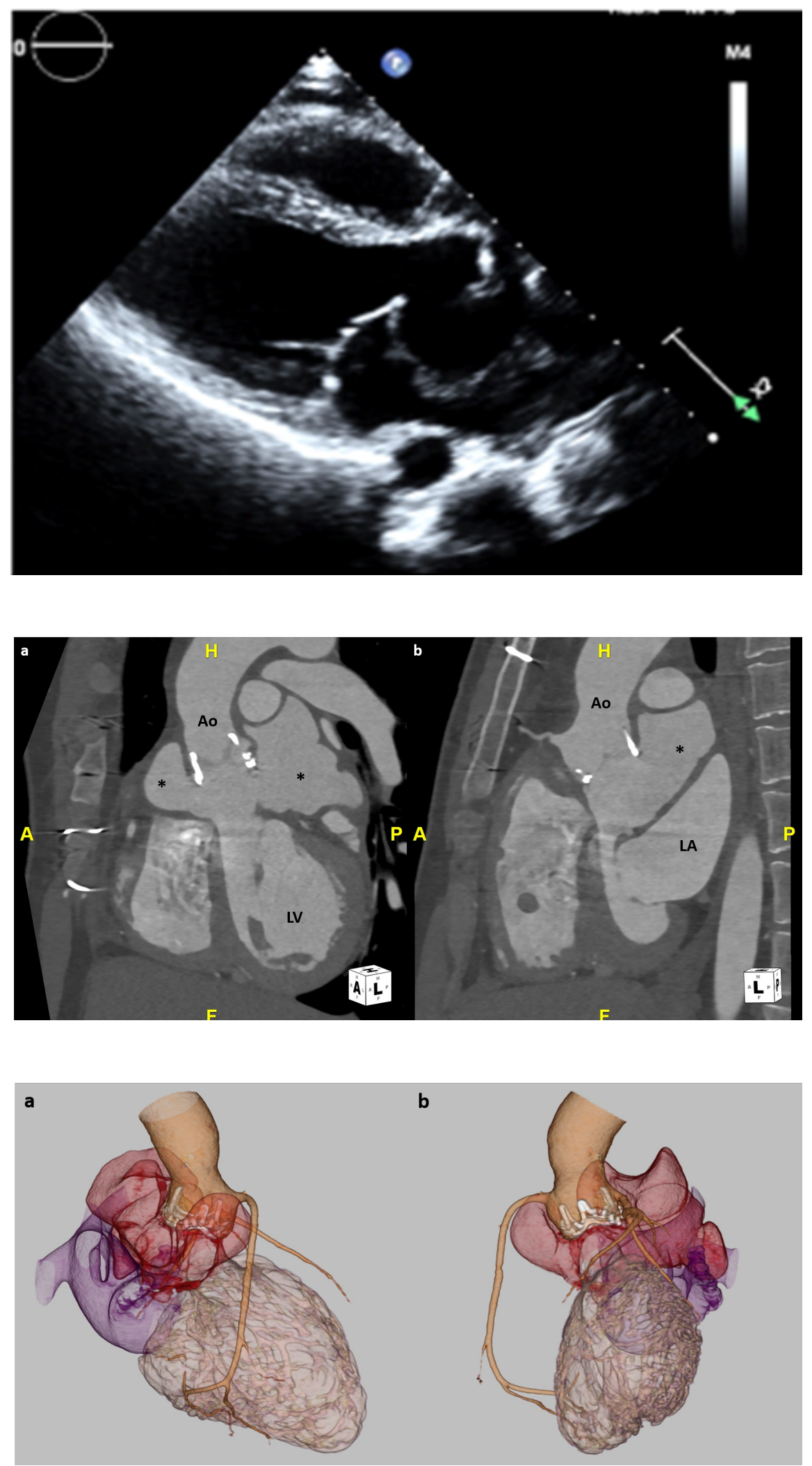


\section{Hosted file}

EXP0000_framesX2_(2).mp4 available at https://authorea.com/users/456014/articles/553221complete-postsurgical-left-ventricular-aortic-discontinuity-and-pseudoaneurysm-formation 